\title{
Cuando la música cesa: el papel de los festivales culturales en la creación de espacio urbano
}

\author{
Soledad Morales Pérez \\ Carmen Pacheco Bernal \\ Universitat Oberta de Catalunya. Estudios de Economía y Empresa \\ smoralespe@uoc.edu \\ mpachecob@uoc.edu
}

Recibido: octubre de 2016

Aceptado: mayo de 2017

\section{Resumen}

El presente trabajo se centra en analizar cómo contribuyen los festivales culturales a la creación de espacio urbano a través del estudio de la percepción que los residentes tienen de los impactos sobre la vida diaria de la ciudad de un festival musical público organizado de manera continuada desde hace más de 28 años, el Mercat de Música Viva de Vic. Mediante un análisis factorial exploratorio llevado a cabo con los datos obtenidos en una encuesta realizada a residentes de la ciudad, se demuestra que este festival influye tanto en la dimensión física como percibida de la ciudad y está favoreciendo la redefinición, expansión y redescubrimiento tanto de la vida social de la ciudad como de su espacio físico. Así mismo, al ser portador de significados nuevos, está provocando nuevas narrativas de la ciudad y nuevos vínculos de los ciudadanos hacia ella.

Palabras clave: espacio urbano; festivales culturales; percepción de los residentes; Mercat de Música Viva de Vic

\section{Resum. Quan la música cessa: el paper dels festivals culturals en la creació d'espai urbà}

Aquest treball se centra a analitzar com contribueixen els festivals culturals en la creació d'espai urbà a través de l'estudi de la percepció que els residents tenen dels impactes sobre la vida diària de la ciutat d'un festival musical públic organitzat de manera continuada des de fa més de 28 anys, el Mercat de Música Viva de Vic. Mitjançant una anàlisi factorial exploratòria portada a terme amb les dades obtingudes en una enquesta feta a residents de la ciutat, es demostra que aquest festival influeix tant en la dimensió física com en la percebuda de la ciutat, i està afavorint la redefinició, l'expansió i el redescobriment tant de la vida social de la ciutat com del seu espai físic. Així mateix, en ser portador de significats nous, està provocant noves narratives de la ciutat i nous vincles dels ciutadans cap a ella.

Paraules clau: espai urbà; festivals culturals; percepció dels residents; Mercat de Música Viva de Vic 
Résumé. Quand la musique cesse : le rôle des festivals culturels dans la création de l'espace urbain

Le présent travail se centre sur l'analyse de la manière dont les festivals culturels contribuent à la création d'espace urbain, grâce à une étude de la perception que les résidents ont des impacts du Mercat de Música Viva de Vic, un festival musical public organisé de façon continuelle depuis plus de 28 ans, sur la vie quotidienne de la ville. À travers une analyse factorielle exploratoire réalisée avec les données d'une enquête auprès des habitants de la ville, nous démontrons que ce festival influence à la fois dans la dimension physique et dans la perception de la ville et qu'il a des répercussions positives sur la redéfinition, l'expansion et la redécouverte de la vie sociale de la ville ainsi quede son espace physique ainsi que, et qu'il est en outreporteur de significations nouvelles, provoquant nouvelles narrations de la ville et de nouveaux liens établis entre les citoyens et cette dernière.

Mots-clés: espace urbain; festivals culturels; perception des résidents; Mercat de Música Viva de Vic

Abstract. When the music stops: The role of cultural festivals in the creation of urban space

This paper analyzes how cultural festivals contribute to the creation of urban space through the study of residents' perceptions regarding the impacts on daily life of a public music festival organized annually for over more than 28 years, the Mercat de Música Viva de Vic in Spain. The results of an exploratory factor analysis using data obtained from a survey conducted among residents show that the festival creates multiple spaces which influence both the physical and the perceived dimensions of the city, while favoring the redefinition, expansion and rediscovery of the city's social life and physical space. Moreover, as a bearer of new meanings, the festival is building new narratives of the city and creating new links between the city and its citizens.

Keywords: urban space; culture festivals; resident perception; Mercat de Música Viva de Vic

\section{Sumario}
1. Introducción
4. Metodología
2. El espacio de los festivales o los
5. Resultados
festivales en el espacio
6. Conclusiones
3. La geografía tensionada del Mercat de
Música Viva de Vic
Referencias bibliográficas

\section{Introducción}

Los eventos y las ciudades han vivido siempre relacionados y, según algunos autores, las ayudan a ser más dinámicas y acogedoras (Richards y Palmer, 2010). Los festivales culturales en general, y los musicales en particular, han tenido un protagonismo destacado en esta relación y desde el pasado siglo XX a esta parte han ido ocupando un espacio cada vez mayor en la vida de las ciudades, hasta convertirse actualmente en uno de los formatos dominantes 
de su paisaje cultural. Las instituciones públicas han sido grandes promotoras de este tipo de eventos, bien a través de su organización directa, bien a través de la creación de políticas culturales, convirtiéndose en uno de los agentes principales de la festivalización de las ciudades (Hitters, 2007). Las estrategias que han motivado su aparición son múltiples; pero, principalmente, los festivales se han utilizado como herramientas para generar un amplio abanico de efectos, entre los que se incluyen la generación de ingresos, de cohesión social y de creación y mejora de la imagen de la ciudad (Dwyer et al., 2000; Faulkner et al., 2000; Higham y Ritchie, 2001; Presbury y Edwards, 2005). Como exponen Gibson y Homan (2004), los gobiernos locales han utilizado los festivales para mediatizar en los efectos culturales de la gentrificación y de la renovación urbana y han convertido los culture-led o festival-led en tendencia de los procesos de regeneración urbana en las ciudades postindustriales (Bailey et al., 2004; Evans, 2005; Hall y Page, 2012). Los gobiernos locales y las instituciones públicas en general se han convertido, por tanto, en prescriptores avanzados en la promoción de este tipo de eventos y en uno de los principales agentes de su florecimiento.

Dado este protagonismo de los agentes públicos, es conveniente preguntarse de qué manera los festivales gestionados por la iniciativa pública contribuyen a la vida urbana, y muy especialmente qué consecuencias tienen sobre la creación de espacio en la ciudad. Hay que tener en cuenta que la política pública se utiliza con frecuencia como un medio para facilitar o restringir el acceso a los espacios públicos, y para privilegiar, muchas veces, los intereses económicos. Pero a pesar de que los gobiernos han visto también en los eventos una herramienta para las estrategias de desarrollo comunitario (O'Sullivan et al., 2009) los enfoques y los marcos metodológicos que se han utilizado para evidenciar las consecuencias sobre el territorio de albergar este tipo de eventos no han permitido entender su verdadero valor, y tal como indica Sassatelli (2015), los impactos que los festivales generan a largo plazo, incluidos los sociales, siguen siendo casi un misterio.

Por otro lado, los festivales organizados en ciudades medias o pequeñas han despertado bastante menos interés entre los académicos que los eventos de grandes dimensiones de las grandes ciudades postindustriales. Los estudios que existen al respecto (Brennan-Horley et al., 2007; Curtis, 2010; Gibson y Connell, 2011 y 2012; Gibson y Davidson, 2004) han puesto de manifiesto que los festivales mejoran la imagen de los destinos más pequeños y generan riqueza económica gracias al turismo que atraen y, aún no teniendo su origen en la tradición local y el patrimonio cultural, pueden desempeñar un papel importante y positivo tanto en el desarrollo de las comunidades como de su economía.

Nuestro trabajo parte de la premisa de que los festivales culturales públicos tienen un papel muy significativo en la vida cotidiana de las ciudades y que, a través de la apropiación de espacios públicos y semipúblicos, favorecen la redefinición, el redescubrimiento y la expansión de la vida social local y de los significados de los lugares (Stevens y Shin, 2014). Argumentamos, además, que es clave estudiar este hecho escuchando las voces de los actores locales (Gotham, 
2005). Nuestro objetivo aquí es explorar cómo un festival organizado por un organismo público en una ciudad mediana del centro de Cataluña, que se organiza de manera continuada desde hace más de 28 años, el Mercat de Música Viva de Vic, funciona como una plataforma de construcción, deconstrucción y reconstrucción de la ciudad y de sus significados a través de la percepción que los residentes tienen de sus impactos sobre la vida diaria de la ciudad.

\section{El espacio de los festivales o los festivales en el espacio}

Según Andrew y Leopold (2013), los eventos tienen que ver con los lugares $y$ las personas en el momento en que las personas visitan ciertos lugares para encontrarse o para formar parte de un evento. No obstante, la capacidad que tienen los eventos de crear un espacio y un tiempo vivo y efímero para la reunión y la relación humana (Getz, 2007) hace que su relación con los lugares no sea ni inocua ni neutra sino, además de compleja, rica. Uno de los elementos que convierten a los eventos particularmente interesantes para la investigación geográfica es el hecho de que crean espacios de experiencia colectiva gracias a interrumpir la «normalidad» o la vida cotidiana, convirtiéndose así en una plataforma para el estudio de la espacialización de la vida social. Parece entonces apropiado preguntarse en qué dimensiones o qué tipo de modificaciones sobre el espacio generan los eventos, y su contribución a la vida urbana; pero, también, indagar incluso en la manera cómo crean espacio. Hacernos estas preguntas sobre eventos promovidos por instituciones públicas que tienen lugar en el espacio público puede ser, además, un ejercicio de democracia y transparencia.

Como apunta Cudny (2014), la geografía se ha interesado por los festivales principalmente por su dimensión social y cultural, a pesar de que muchas de las dinámicas generadas por ellos tienen una gran significación geográfica y espacial. Hoy en día, gracias al nuevo marco ontológico generado en las ciencias sociales, especialmente desde aquellos enfoques que nacen en torno a los debates postmodernos, el postestructuralismo y las epistemologías feministas, se ha creado una nueva gramática propicia para (re)pensar la dimensión espacial de los eventos y replantearse preguntas esenciales al respecto de su naturaleza como fenómenos geográficos y su relación con el espacio público. Si el espacio se define como una compleja construcción social que forma las percepciones y las prácticas espaciales, basadas en valores y en la producción social de significados (Rodó-de-Zárate, 2013) y es, como indicaba Lefebvre (2013), al mismo tiempo una precondición y un producto de las superestructuras sociales, no parece ilusorio considerar que el espacio no es solamente el soporte para que un evento tenga lugar, ni los eventos ocurren sin ninguna consecuencia sobre ese espacio. Parecer ser mucho más adecuado pensar esta conexión más bien como una relación dialógica que puede ser o no efímera.

En este sentido, Quinn (2006), por ejemplo, apunta que los festivales «tienen una fuerte dimensión colectiva y siempre han funcionado como prácticas a partir de las cuales las comunidades han expresados creencias, celebran 
identidades y confirman o contestan de manera diversa las estructuras sociales y los sistemas de valores que las unen" (pág. 289). Parece entonces que los festivales ofrecen oportunidades únicas para que las personas se relacionen de manera diferente pero también refuercen las relaciones y los lazos sociales preexistentes (Gibson y Connell, 2011) y faciliten la relación social a un escala local (Stevens y Shin, 2014). Diversos autores reconocen que los eventos, además de contribuir al desarrollo social y cultural, crean y comunican valores sociales y potencian el intercambio social, el fortalecimiento de la identidad y cultura local o el fomento del sentimiento de orgullo y pertenencia a la comunidad local (Carlsen et al., 2007; De Bres y Davis, 2001; Moscardo, 2007; Small, 2008). Tanto es así, que Derret (2003) asegura que los festivales actúan no solamente como atractivos turísticos sino también como una cultural fabric of a place. Es decir, los eventos juegan un papel muy relevante en la conexión de las personas con los lugares y en la creación de significados y las narrativas subjetivas alrededor de ellos, con lo que son parte de la puesta en escena de los eventos y estos, al mismo tiempo, dan forma y definen (Getz y Page, 2015) los lugares que los albergan.

Según Thrift (2006), la incorporación de la teoría de la performatividad en geografía ha generado un resurgimiento del espacio como concepto, un nuevo materialismo, que ha servido para repensar el espacio y moverlo desde concepciones binarias y absolutistas (newtonianas y kantianas) hacia términos relacionales que remarcan la liquidez del espacio y de las prácticas que se superponen a él dentro de entornos que ahora se suponen cocreados o coconstruidos dentro de límites porosos (Jones, 2009). Un nuevo paradigma espacial que considera que el espacio se construye a través de sus relaciones y ha dejado de ser un mero escenario en el que se localizan fenómenos (Villarino, 2012). Si para el turismo el giro performativo ha servido (además de para posicionar la disciplina desde los márgenes hasta el centro de las ciencias sociales) para interesarse no ya sobre lo que le pasa al turismo sino lo que sucede en torno a él (Russo, 2013), este nuevo marco ontológico comporta también la necesidad de una mayor interacción entre disciplinas (Malpas, 2015).

Esta nueva ontología relacional y performativa nacida de la reacción al absolutismo espacial y a su conceptualización como «realidad dada» nos habla de la naturaleza del espacio y reconoce que los lugares se viven, toman vida y se transforman con su uso, y son las prácticas situadas, las acciones y las interacciones sociales los elementos que pueden dibujar un nuevo significado del espacio (Larsen, 2011). El espacio es, por tanto, relacional pero también usado, experimentado y toma significado alrededor de estos dos elementos. De esta forma, los autores que han abrazado la perspectiva basada en la performatividad y la performance le adscriben una importancia esencial a la relacionalidad y los encuentros, en la que la relacionalidad se refiere a todas las interacciones a través de las cuales las entidades llegan a existir, y las incluye. En este sentido, los eventos crean las condiciones, el teatro, gracias a las cuales los lugares toman vida y se transforman; las personas (sean residentes o visitantes) se relacionan, y su performatividad sobre el espacio le generan y proyectan nuevos significados. 
Son espacios (o pueden ser espacios) a través de los cuales los lugares se pueden experimentar desde un punto de vista multisensorial y pueden dibujar una geografía emocional de la sociabilidad. Estudios como los de Lucas y Wright (2013) o Giovanardi et al. (2014) son exponentes de ello. Estos últimos, por ejemplo, llegan a profundizar en el carácter interrelacional e intersubjetivo de la performatividad a través del estudio de las relaciones entre turistas, residentes y trabajadores turísticos en la celebración de un evento, contestando las visiones binarias que confrontaban residentes y turistas y mostrando cómo un lugar turístico es cocreado por los diferentes agentes que intervienen en él.

\subsection{Festivales públicos, residentes y geometrías de poder}

Con el giro performativo «la idea de espacio público comienza a elaborarse en términos de práctica, proceso, capacidad, acontecimiento o posibilidad» (Villarino, 2012, pág. 7) y toma sentido hablar de él en función de sus rutinas de ocupación y uso y lo que sucede alrededor de ellas. En este sentido, Carmona (2015) considera además que, actualmente, es necesaria una visión del espacio público que reconozca los múltiples tipos, papeles y usuarios de los espacios públicos de la ciudades de hoy en día, dado que es demasiado utópica la visión que define el espacio público como «an open and inclusive stage for social interaction, political action and cultural exchange» (pág. 376). Esta visión del espacio público como una arena de relaciones tensionadas, formada por multitud de prácticas de contestación y negociación cotidiana, exige de su politización, tal como postulaba Massey (2005) y como han puesto de manifiesto sobradamente los enfoques feministas o aquellos que han querido hacer visibles las alteridades y las desigualdades (Markwell y Waitt, 2009; Ortiz et al., 2014 y Waitt y Stapel, 2011).

Dredge y Whitford (2011) dicen al respecto del espacio público que es la arena para la democracia participativa y discursiva, el espacio en el que tiene lugar la gobernanza de los lugares. Parece entonces interesante poner uno de los focos de atención de los estudios sobre eventos y creación de ciudad en los residentes y en sus percepciones sobre los efectos que estos tienen sobre su vida diaria. Centrar el interés en las percepciones de los residentes de un lugar y darles voz no ha sido una práctica excesivamente habitual en el estudio de los festivales pero no así en el campo turístico, como lo demuestra el estudio de Deery et al. (2012) o por ejemplo los trabajos de Ap (1992) o Sharpley (2014). Esto ha significado perder una información muy rica y relevante tanto para las administraciones públicas como para los organizadores de estos eventos, ya que los residentes viven en primera persona y en su vida cotidiana los impactos de los eventos que se organizan en su lugar de residencia.

Se podría decir, incluso, que los residentes tienen un derecho moral adquirido para ser escuchados, más aún si tenemos en cuenta que ponen al servicio de los demás recursos que son propios y que forman parte de su vida diaria. De hecho, las instituciones podrían ser sensibles y astutas para utilizar este conocimiento y la experiencia que acumulan los residentes a este 
respecto (el de los impactos de los eventos) para gestionar de una manera eficaz las áreas de interés local (Weaver y Lawton, 2013). Y para finalizar, los residentes son los que directamente interactúan con los visitantes foráneos y por tanto tienen el poder de hacer prosperar el turismo o los eventos o bien resistirse a ellos, ya que la insatisfacción de una comunidad puede amenazar el éxito del evento a largo plazo aunque sea económicamente viable (Small et al., 2005).

Es porque el espacio no es un objeto científico descarriado de la ideología o de la política (Lefebvre, 2013) que la consideración del espacio público no puede considerarse neutra en relación con las geometrías del poder ni exenta de tensiones. Un poder que se distribuye, eso sí, de manera ubicua y se ejerce en todas las relaciones y en todas las direcciones. De hecho, existen autores a favor y en contra de la proliferación de festivales en las ciudades y en sus espacios públicos, porque su existencia tiene que ver y se relaciona con las estructuras de poder y los discursos políticos determinados. Gotham (2005), utilizando las ideas de Guy Debord y Henri Lefebvre, indica que los festivales se han convertido en campos de batalla de contención y se organizan por diferentes grupos con intereses diversos que intentan producirlos para sus propios fines. David Harvey, por ejemplo, considera que «the mass production of festivals and celebrations creates 'voodoo cities' in which the facade of cultural redevelopment can be seen as a 'carnival mask' that covers continuing disinvestment and increasing social inequality» (1988, pág. 168, 2000), (citado en Gotham, 2005).

Es cierto que la globalización, la comunicación de masas y la movilidad global han conllevado la pérdida de autenticidad e incluso identidad de los lugares; un placelessness producido por la homogeneización que conlleva el capitalismo global. Pero los festivales, entendidos como espacios únicos e irrepetibles que difieren de un hecho cotidiano, que se experimentan solamente si se forma parte de ellos, también pueden ser, tal como exponen Lucas y Wright (2013), espacios de transición (liminales) marcados por la ambigüedad o la desorientación, en los que el orden y las jerarquías establecidas pueden disolverse temporalmente o transformarse. Pueden ser contextos en los que las identidades se cuestionen o se cambien, en los que las posiciones subjetivas no se sostengan y los actores puedan adoptar identidades de manera temporal que pueden o no convertirse en permanentes. Como el mismo Gotham identifica (2005), existen también actores locales que usan los festivales como altavoces para acciones reflexivas de críticas radicales sobre la desigualdad. Sharpe (2008) incluso sostiene que algunos festivales pueden (re)conectar el ocio con la acción política y el cambio social en formas significativas y eficaces que son especialmente importantes para la comunidad local a través de nuevas formas de pleasure-politic. En definitiva, la observación y el análisis de las geometrías del poder en torno a los eventos nos pueden dar claves para entender cómo el espacio es negociado y reconfigurado o incluso sobre la construcción política de los espacios de vida. 


\section{La geografía tensionada del Mercat de Música Viva de Vic}

Vic es una ciudad de 41.956 habitantes (<www.idescat.cat $>$ ), situada en el centro de Cataluña. El Mercat de Música Viva de Vic (MMVV) (<www. mmvv.cat>) es uno de los eventos con mayor proyección de todos los que se organizan desde la ciudad y ha hecho que la música, sin ser uno de los rasgos identitarios de la historia de Vic, se haya convertido en uno de los iconos de la ciudad actual. El MMVV es, además, un evento marcadamente público, generado y gestionado principalmente por el Ajuntament de Vic a través del Organisme Autònom de Fires i Mercats de Vic (OFIM) (<www.vicfires.cat>), con el apoyo directo del Institut Català de les Empreses Culturals (ICEC) de la Generalitat de Catalunya y de otras instituciones públicas catalanas y estatales. Como muchos otros festivales de otras industrias culturales, el MMVV es la combinación de dos tipos de eventos: un festival musical con una oferta de conciertos (la mayoría de ellos gratuitos) dirigidos al público en general y una feria profesional conformada por un espacio específico para los profesionales de la industria de la música (un Mercat de música, como su nombre indica). Estas dos dimensiones del festival se unen y proyectan, y también se tensionan en el espacio urbano y lo llegan a convertir en un festival intensivo en el uso, consumo y en la modificación del espacio urbano, con capacidad para redefinirlo, otorgarle una identidad temporalmente nueva y enfatizar su función colectiva, pero también para mercantilizarlo y asociarlo con la industria cultural contemporánea y sus fundamentos comerciales.

La distribución geográfica de los lugares públicos en los que se programan los conciertos del MMVV responde a una lógica estratégica que tiene en cuenta tanto los diferentes perfiles de público (asistentes y profesionales) como los diversos estilos de música que se programan. La Plaça del Mercat o Plaça Major, un recinto medieval de gran autenticidad y simbolismo para la ciudad, está asociada a la dimensión pública del evento y es una de las imágenes más conocidas del MMVV. En este epicentro se programan los conciertos de mayor expectación para el gran público y no es difícil ver a familias mezcladas entre la audiencia. Pero también es el lugar en el que más se proyectan y al mismo tiempo proyecta las representaciones de la identidad y del sentido del lugar, como por ejemplo las vinculadas a la identidad catalana y sus procesos políticos.

En el vértice sur de la ciudad, en cambio, el espacio público y el festival se privatizan, se utilizan infraestructuras públicas para conciertos de pago y se construyen estructuras efímeras (carpas) para facilitar la dimensión comercial del evento. El espacio se ordena en función de diversos estilos musicales, lo que ha permitido a la organización crear un producto comercializable para el público asistente y diseñar itinerarios para la mejor visualización de música para los profesionales. El MMVV es un evento posttradicional que no está enraizado del todo en el desarrollo orgánico de la cultura del lugar, sino que está asociado con la industria cultural contemporánea y sus fundamentos. Es precisamente la crítica a esta dimensión del festival y a la vinculación de las 
instituciones públicas y las políticas culturales con las industrias culturales y a su desconexión con el ecosistema musical local, la que ha generado la concatenación y superposición de eventos y expresiones artísticas paralelas e independientes o semiindependientes a las acciones del MMVV en el espacio público. Estas contraprogramaciones o festival Off se ordenan alrededor de tres propuestas concretas: el Sugar (Il-legal) Fest; el Festival Hoteler y el Escenari Muralla, algunos de ellos herederos de otro festival Off del Mercat, ya extinguido, el InVICtro.

Estas contraprogramaciones son capaces de crear un discurso político alrededor de la música y proyectarlo, críticamente, a través de su performatividad en espacio público de la ciudad con expresiones disruptivas y cuestionadoras del orden social existente y la normatividad, y no sólo las de las tradiciones o de la identidad de otros sectores sociales sino de la manera como se gestiona el negocio de la música. De hecho, son capaces de crear espacios críticos de participación musical o alrededor de la música, al margen del propio Mercat (aunque algunos de ellos, como el Festival Hoteler, con una cierta proximidad a él), capaces de dar visibilidad a la identidad y el ecosistema cultural de la región y a su cultura de base más underground. En definitiva, la propuestas Off que nacen alrededor del MMVV son generadoras de paisajes culturales extraordinarios, fuera de la normatividad, como es el caso del Sugar (Il-legal) Fest, con respecto a una identidad cultural y un posicionamiento político (en contraposición) con el discurso normativo representado, según ellos, por el MMVV. Estas manifestaciones culturales coexisten de manera líquida y permeable con los espacios generados por el MMVV; pero, al mismo tiempo, evidencian las tensiones y las relaciones de poder que se proyectan sobre el espacio público. Su puesta en escena está vinculada a la intencionalidad artística que implica un deseo de ser visto o escuchado, lo que al mismo tiempo supone un proceso de comunicación y representación. Son una fuente que incrementa el capital creativo que rodea a un evento de carácter público como el MMVV y que ejemplifican, aún más, la complejidad de las experiencias que se pueden generar alrededor y sobre el espacio público y las tensiones que constatan, incluso, las estructuras de poder y la contestación a las mismas.

\section{Metodología}

Para el estudio que presentamos se ha utilizado la triangulación metodológica y el análisis factorial exploratorio de los resultados obtenidos de una encuesta dirigida a residentes de la ciudad. Las encuestas fueron administradas telefónicamente a residentes con domicilio en el Barri del Centre de la ciudad, es decir, con residencia en los lugares - o cerca de ellos- en los que se instalan los escenarios del evento. El trabajo de campo se realizó la semana después de la finalización de la edición de 2012 del MMVV y se recogieron un total de 139 respuestas válidas, de las cuales el 52,5\% provenían de hombres y el $47,5 \%$, de mujeres, con una media de edad de 47,3 años. 
El núcleo del cuestionario constaba de 29 que tenían que ver con seis factores (dimensiones) de los impactos del evento sobre la ciudad y la vida cotidiana de los residentes, a saber: la económica, turismo/imagen, urbanística, sociocultural, social-psicológica y ambiental. Previamente al lanzamiento de la encuesta, se llevaron a cabo cinco entrevistas en profundidad a responsables tanto de la organización del evento como de la gestión de la ciudad. Estas entrevistas semiestructuradas nos sirvieron además para entender los objetivos estratégicos del evento y sus efectos sobre las diferentes dimensiones de la ciudad, así como para diseñar el cuestionario destinado a los residentes de la ciudad y para interpretar los resultados del trabajo de campo cuantitativo.

Las escalas de medida utilizadas en el cuestionario para evaluar el valor del impacto del evento se adaptaron de Small et al. (2005), de Fredline et al. (2003) y de Delamere et al. (2001). Los ítems se debían valorar en una escala de Likert de 7 puntos, donde 1 significaba «Totalmente en desacuerdo" y 7 «Totalmente de acuerdo» con la afirmación propuesta.

\section{Resultados}

Atendiendo a las puntuaciones medias obtenidas en la escala 1-7 por cada ítem (tabla 1), podemos afirmar que los residentes consideran que el MMVV tiene una influencia principalmente positiva sobre la ciudad. Sin embargo, la valoración del festival por parte de los residentes no está exenta de elementos negativos o conflictivos y pone de manifiesto las posibles tensiones que la organización de un evento en el espacio público de la ciudad puede generar entre la ciudadanía y en el uso del mismo. No obstante, a pesar de reconocer la existencia de impactos negativos del MMVV, los ciudadanos no los perciben lo suficientemente relevantes como para generar una actitud de rechazo hacia el evento. Al contrario, los ciudadanos de Vic expresan un mayor grado de acuerdo con aquellos ítems que muestran una actitud positiva hacia el evento, tanto en aspectos colectivos como individuales, principalmente pertenecientes a la dimensión del valor y los impactos sociales del evento, como es la generación de capital social y el sentimiento de orgullo y pertenencia, y que se resumen en los siguientes ítems: «El MMVV me da la oportunidad de disfrutar con los amigos» (5,58), «El MMVV ha hecho que los músicos catalanes tengan más oportunidades» $(5,40)$, «El MMVV transmite una buena imagen de Vic» $(5,34)$, «El MMVV incrementa las oportunidades para conocer nuevas personas» $(5,25)$, «El MMVV incrementa el orgullo que los ciudadanos tienen de Vic» $(5,20)$, "El MMVV promueve las interacciones/relación entre personas locales y foráneas» $(5,20)$.

Con el objetivo de explorar las relaciones entre los ítems testados, y obtener factores que identificaran constructos subyacentes, se llevó a cabo un análisis factorial exploratorio. El resultado de dicho análisis estadístico nos permite obtener los factores que determinan la percepción de los residentes de Vic respecto al MMVV y dar respuesta a la pregunta de investigación que nos hemos formulado sobre el papel que un festival público puede tener en la construc- 
Tabla 1. Resultados del análisis factorial. Puntuaciones factoriales de las variables en cada factor y media (escala 1-7 de valoración) obtenida de cada variable

\begin{tabular}{|c|c|c|c|c|}
\hline \multirow[b]{2}{*}{ Media (1-7) } & \multicolumn{3}{|c|}{ Factores } & \multirow[b]{2}{*}{ Factor 1: Identidad, cohesión social y proyección } \\
\hline & 1 & 2 & 3 & \\
\hline 5,34 & ,755 & ,254 & 279 & El MMVV transmite una buena imagen de Vic \\
\hline 4,92 & ,689 & ,257 & ,211 & El MMVV promueve valores que son buenos \\
\hline 4,71 & ,685 &, 141 &, 127 & El MMVV ha hecho de la música un eje central de la cultura en Vic \\
\hline 5,03 & ,665 & ,296 &, 044 & El MMVV ha hecho que Vic se proyecte al mundo \\
\hline 5,08 & ,662 &, 344 &, 156 & El MMVV incrementa y mejora la reputación de Vic \\
\hline 5,17 & ,631 & ,279 &, 010 & El MMVV amplía las oportunidades de ocio en la ciudad \\
\hline 5,58 & ,626 & ,213 & 197 & El MMVV me da la oportunidad de disfrutar con los amigos \\
\hline 4,58 &, 588 & ,325 & ,220 & El MMVV da la oportunidad de enseñar a los demás la cultura vigatana \\
\hline 5,20 & ,588 &, 419 & ,205 & El MMVV incrementa el orgullo que los ciudadanos tienen de Vic \\
\hline 5,25 &, 575 &, 155 &, 029 & El MMVV incrementa las oportunidades para conocer nuevas personas \\
\hline 5,18 & ,541 & ,345 &,- 073 & Gracias a aparecer en los medios, el MMVV promueve el turismo y los negocios en Vic \\
\hline 5,40 & ,464 &,- 089 & ,356 & El MMVV ha hecho que los músicos catalanes tengan más oportunidades \\
\hline \multirow[t]{2}{*}{4,09} & ,382 &,- 062 &,- 039 & El MMVV ha hecho incrementar la disponibilidad de aparcamiento en las inmediaciones \\
\hline & & & & Factor 2: Dimensión económica y urbanística del evento \\
\hline 4,39 & ,252 &, 717 &, 010 & Gracias al MMV, la población de Vic goza de más instalaciones disponibles \\
\hline 4,15 &,- 045 & ,698 & ,173 & El MMVV amplía los derechos y las libertades civiles de los residentes \\
\hline 4,81 &, 043 & ,628 &,- 074 & El MMVV genera puestos de trabajo \\
\hline 4,47 & ,324 & ,587 &, 178 & El MMVV ayuda al mantenimiento de las áreas cercanas al evento \\
\hline 4,24 & ,336 & ,566 &, 146 & El MMVV ayuda al mantenimiento de los servicios públicos \\
\hline 4,97 &, 410 & ,562 &,- 066 & Gracias al dinero que se gastan los turistas y los visitantes se estimula la economía de Vic \\
\hline 5,09 & ,333 & ,548 &,- 198 & El MMVV hace incrementar los ingresos de los negocios locales \\
\hline 5,01 &, 453 &, 535 & ,221 & El MMVV crea comunidad \\
\hline \multirow[t]{2}{*}{5,20} & ,312 &, 474 & ,459 & El MMVV promueve las interacciones/relación entre personas locales y foráneas \\
\hline & & & & Factor 3: Aspectos negativos con impacto ambiental y económico \\
\hline 4,08 &, 061 & ,005 &, 741 & El MMVV incrementa la desigualdad social porque beneficia a los ricos y no a los pobres \\
\hline 3,88 &, 161 &,- 002 &, 731 & El MMVV genera comportamientos violentos y delincuencia \\
\hline 4,01 &,- 192 &, 183 & ,674 & El MMVV ha modificado el coste de la vida en general \\
\hline 3,95 &,- 123 & ,130 & 671 & El MMVV ha modificado los precios de algunos bienes y servicios \\
\hline 3,80 & ,201 &, 086 & ,615 & El MMVV interrumpe la vida de los vigatanos y les provoca estrés \\
\hline 3,40 & ,305 &,- 026 &, 562 & El MMVV provoca que haya más basura en los alrededores \\
\hline 3,68 & ,267 &,- 089 &, 537 & El MMVV genera congestiones de tráfico en las inmediaciones \\
\hline $\begin{array}{l}\text { Alfa de } \\
\text { Cronbach }\end{array}$ & ,896 &, 844 &, 779 & \\
\hline $\begin{array}{l}\text { \%Varianza } \\
\text { explicada } \\
\text { (total=48,2\%) }\end{array}$ & 30,8 & 11,0 & 6,3 & \\
\hline
\end{tabular}

Método de extracción: Análisis de componentes principales.

Método de rotación: Normalización Varimax con Kaiser.

Fuente: elaboración propia. 
ción y configuración de la ciudad y de sus significados, según la percepción que los residentes tienen de sus impactos sobre la vida cotidiana de la ciudad.

La interpretación de los resultados que nos proporciona el análisis factorial exploratorio permite identificar tres factores, obtenidos a partir de las 29 variables iniciales, que explican el $48,12 \%$ de la varianza total. La tabla 1 recoge los resultados del análisis. El primer factor (que explica el 30,8\% de la varianza total) está relacionado con la función social del evento y con factores socialmente construidos e intangibles con influencia en la subjetividad y los significados proyectados sobre la ciudad, dado que la mayoría de los 13 ítems que resumen este factor apuntan actitudes favorables hacia estos aspectos por parte de los ciudadanos residentes en Vic. El segundo factor (que recoge el 11,0\% de la varianza total) describe percepciones relacionadas con la dimensión económica y urbanística del evento, mientras que el tercero (6,3\% de la varianza total) presenta las percepciones sobre los aspectos más negativos que tienen más que ver con los impactos ambientales y económicos en la ciudad.

Posteriormente, se procedió al análisis de las diferencias de sexo y de edad que podían afectar estos resultados. Según estos, la variable de sexo — para nuestro caso de estudio - no introdujo cambios significativos ni en la percepción de los impactos sobre el territorio ni su distribución; pero no fue así para la variable generacional. Los residentes más jóvenes destacaron aún más que el resto de entrevistados los aspectos de capital social vinculados con el redescubrimiento y la expansión de la vida social, como por ejemplo los ítems relacionados con el bonding social capital ( $\mathrm{El}$ MMVV me da la oportunidad de disfrutar con los amigos») y el bridging social capital («El MMVV incrementa las oportunidades de conocer a otras personas»), así como de creación de comunidad («El MMVV crea comunidad»).

\subsection{El espacio social y las nuevas narrativas de la ciudad alrededor del $M M V V$}

El primer factor identificado en el análisis factorial exploratorio nos ha permitido agrupar aquellos elementos del MMVV que tienen, según la percepción de los ciudadanos, un impacto mayor y más positivo sobre la ciudad y sobre su vida cotidiana. En este primer factor se agrupan un gran número de ítems con los que se demuestra que los residentes de Vic reconocen la capacidad del festival para generar un espacio lleno de tejido y de intercambio social, de performatividad colectiva, que influencia positivamente la cohesión del entramado social de la ciudad. El mapa de interrelaciones que se dibuja gracias a la participación en el MMVV tanto entre residentes (amigos) y visitantes/ turistas (en menor medida) permite ver que uno de los efectos más evidentes de la organización de este evento es la relacionalidad y la creación de capital social, a través del cual los ciudadanos perciben un fortalecimiento no sólo de sus relaciones sino de su sentimiento de orgullo y de pertenencia a la comunidad local. Es en este momento en el que los impactos percibidos del MMVV profundizan en las dimensiones psicosociales y socioculturales del espacio. 
Los ítems pertenecientes a este primer conjunto de percepciones que muestran un mayor grado de acuerdo son «El MMVV me da la oportunidad de disfrutar con los amigos» $(5,58)$, «El MMVV transmite una buena imagen de Vic» (5,34), «El MMVV incrementa las oportunidades para conocer nuevas personas» $(5,25), \mathrm{y}$ «El MMVV incrementa el orgullo que los ciudadanos tienen de Vic» $(5,20)$.

En este primer factor aparecen además elementos que tienen que ver también con la influencia del festival en la misma significación de la ciudad, en su identidad y proyección cultural y en su posicionamiento relativo sobre el territorio. El hecho de que el MMVV genere, a los ojos y la opinión de los ciudadanos, una buena imagen de la ciudad $(5,34)$, junto con la mejora y el incremento de la reputación $(5,08)$ y su proyección hacia el exterior $(5,03)$ y los efectos positivos que este tiene sobre la atracción turística $(5,18)$, pone de manifiesto su influencia sobre la construcción de la imagen (mejorada gracias a su asociación con la cultura, en general, y con la música en particular) de la ciudad y de nuevas narrativas sobre ella. Los ciudadanos han interiorizado y positivizado las narrativas que asocian a Vic con la cultura y con la modernidad de la música y reconstruyen su identidad en base a valores nuevos que parecen volatilizar las visiones costumbristas del territorio en el que se enmarca. Como si Vic, gracias a los valores que le proyecta el MMVV, dejase de ser, no ya temporalmente, una ciudad de «fires i firetes» para pasar a ser una ciudad proyectada al mundo de manera renovada y reconstruida.

\subsection{Los efectos del $M M V V$ sobre la dimensión económica y urbanistica de la ciudad}

El segundo factor resume las percepciones de los ciudadanos de Vic asociadas a la generación de impactos sobre la economía y el urbanismo local. Se trata de percepciones vinculadas a aquellos aspectos en los que el evento influye principalmente en la construcción física de la ciudad, focalizada en la dinamización económica y la generación de riqueza material (dimensión económica) o en la creación de espacio físico a través de la creación o mantenimiento de infraestructuras o servicios (dimensión urbanística).

Las percepciones vinculadas a esta dimensión económica y urbanística que alcanzan un mayor grado de acuerdo entre los residentes son: «El MMVV hace incrementar los ingresos de los negocios locales» $(5,09)$, «Gracias al dinero que se gastan los turistas y los visitantes se estimula la economía de Vic» $(4,97)$, «El MMVV genera puestos de trabajo» (4,81), y «Gracias al MMVV, la población de Vic goza de más instalaciones disponibles» $(4,39)$.

En esta segunda dimensión, los ciudadanos reconocen que el MMVV genera una influencia positiva sobre el dinamismo, principalmente comercial y también turístico (estrechamente relacionados en el caso de Vic), de la ciudad (que se concentra en los días de festival gracias a la atracción de público que genera) y le aceptan ser fuente de estímulo de la economía de Vic (principalmente por la importancia del comercio en su estructura económica). No obstante, no se 
identifica que su influencia se proyecte sobre el sector cultural de la ciudad aunque sí, y de manera muy generalizada, se le presupone una influencia positiva sobre la música catalana en general («El MMVV ha hecho que los músicos catalanes tengan más oportunidades»). Este hecho nos lleva a pensar que la influencia económica del MMVV (pretendida estratégicamente por el mismo festival y las políticas culturales) se sitúa, no en la escena cultural local, sino en los circuitos comerciales de la industria musical y cultural, convirtiéndose en un agente más de los procesos de mercantilización de la música y la cultura de la economía de mercado y alejándose de las posibles necesidades e identidades culturales más arraigadas al territorio.

Se identifica también la influencia del festival sobre la disponibilidad y mantenimiento de servicios e instalaciones públicas a las que la población local tiene acceso. Las valoraciones de este impacto son positivas y similares; pero es relevante el grado de acuerdo, especialmente para la primera de las dinámicas. El hecho de generar este nivel de acuerdo nos puede llevar a pensar que el MMVV, dada su prolongada existencia, se ha convertido en un elemento constitutivo de la vida cotidiana de la ciudad y los residentes lo conciben como un elemento estructural de las propuestas culturales públicas de la ciudad, y a ella lo asocian. De hecho, este evento se proyecta como un usuario privilegiado de la ciudad y sus recursos; pero, al mismo tiempo, un promotor justificativo de las inversiones que las instituciones públicas pueden hacer sobre la ciudad. Le dan sentido y justifican, posiblemente, su existencia.

\subsection{Espacios de tensión y conflicto alrededor del $M M V V$}

El tercer factor que se proyecta del análisis factorial exploratorio recoge aquellas variables relacionadas con el impacto negativo que los ciudadanos de Vic perciben del evento y las interrupciones que genera sobre la vida cotidiana y sobre el uso de los espacios públicos. En este sentido, ponen en evidencia las contradicciones y las tensiones y conflictos surgidos alrededor del festival, contrapuestos a las percepciones positivas analizadas con anterioridad.

Los elementos que más destacan en esta dimensión son «El MMVV genera comportamientos violentos» $(3,88)$, «El MMVV interrumpe la vida de los vigatanos y les provoca estrés» $(3,80)$, «El MMVV genera congestiones de tráfico en las inmediaciones» $(3,68)$ y «El MMVV provoca que haya más basura en los alrededores» $(3,40)$. Existen también otros elementos negativos vinculados más con las consecuencias que el MMVV puede tener sobre la equidad y la justicia social en la ciudad. Las percepciones vinculadas a este respecto han obtenido las siguientes puntuaciones medias en cuanto al grado de acuerdo con las mismas: «El MMVV incrementa la desigualdad social porque beneficia a los ricos y no a los pobres» (4,08), «El MMVV ha modificado el coste de la vida en general» $(4,01)$, $y$ «El MMVV ha modificado los precios de algunos bienes y servicios", $(3,95)$.

A pesar de que estos factores no demuestran un rechazo del evento, sí ponen en evidencia tensiones y conflictos generados en la ciudad por los com- 
portamientos (o performatividades) que se proyectan en los espacios del festival y por el posible colapso del espacio público. Si bien el MMVV no parece crear distorsiones en la estructura social de la ciudad (más al contrario), en ciertos aspectos interrumpe e incomoda la vida cotidiana de los residentes y es escenario de comportamientos marginales. Pero lo que es más, según la percepción de la población consultada, no es un agente que favorezca la redistribución de los recursos o revierta las estructuras sociales, ya que no tiene un papel destacado ni en la gobernanza ni en el freno de los efectos del consumo capitalista de bienes y servicios. En este caso parece ser que el festival es más una plataforma para difundir narraciones legitimizadas, autorizadas y "pacificadoras» del lugar. Y todavía más, el impacto ambiental que genera sobre la ciudad es gestionado por los propios servicios públicos y, a pesar de ser una preocupación para los organizadores, no ha sido hasta la edición de 2017 que se han introducido medidas serias para reducir el impacto del consumo de plástico.

\section{Conclusiones}

Este trabajo ha perseguido el objetivo de contribuir de manera exploratoria a la comprensión de los efectos que los festivales organizados por organismos públicos de manera recurrente tienen sobre la creación de espacio en la ciudad. Hemos estudiado el caso de Vic, una ciudad de tamaño medio que sin tener en la música una de sus señales principales de identidad ha creado, por iniciativa y con recursos públicos, un festival musical, el Mercat de Música Viva, que cuenta ya con 28 años de existencia, y lo ha integrado plenamente en la vida de la ciudad.

A la luz de los resultados del estudio, podemos decir que el MMVV es un agente que interacciona tanto con la dimensión física como percibida de la ciudad. Además, estos resultados ponen en evidencia que el espacio no es estático, sino que se moldea y reformula de manera continuada gracias a la acción de diferentes actores, entre los que cabe incluir los festivales culturales y los agentes asociados a ellos. Se podría afirmar en este sentido que los festivales llenan los espacios urbanos, generan las condiciones para que los lugares "tengan lugar» y se apropian de ellos.

Las percepciones de los residentes demuestran que el MMVV contribuye a la construcción social del espacio y ayuda a mantener su sistema social de diversas maneras, especialmente a través del fomento y la creación de redes de relaciones. Es decir, el MMVV — gracias a la sociabilidad informal y a la convivencia circunstancial que se genera a su alrededor - llena el espacio de tejido social, de performaciones individuales y colectivas y de espacios de intercambio. Este fomento de la relacionalidad se experimenta principalmente entre residentes, pero también se amplía hacia visitantes o turistas, rompiéndose la dicotomía (incluso el enfrentamiento) entre unos y otros. En este sentido, y teniendo en cuenta el nuevo giro ontológico en geografía, sería interesante para futuros trabajos profundizar en la performatividad de estas relaciones (Russo, 2013) y fijar la atención, como indica Popke (citado en Villarino, 2012), «en los cuer- 
pos, las miradas, la comunicación no-verbal, lo prerreflexivo, las interacciones (sean o no focalizadas), etc., pues no sólo son los lenguajes vehiculares de los espacios públicos, sino que también constituyen su ontología contemporánea» (p. 158). De esta manera, se podría profundizar en cómo los ciudadanos se apropian del espacio público, de sus prácticas sobre él; pero también las formas que adquiere esta nueva socialización líquida que se ha identificado.

Como indicaban Stevens y Shin (2014), el MMVV, además, está favoreciendo la redefinición, expansión y redescubrimiento de la vida social de Vic $y$, al ser portador de significados nuevos, está provocando nuevas narrativas de la ciudad, nuevos vínculos de los ciudadanos hacia ella y nuevos espacios de representación (Lefebvre, 2013). Los espacios públicos, nuevos o reconfigurados, adquieren significado en base a las relaciones sociales y a las experiencias colectivas e individuales que se generan alrededor del MMVV, pero también a los asociados a la música. Es en este sentido que la dimensión geográfica del MMVV da claves sobre de qué manera los festivales pueden facilitar la relación social a escala local, cómo crean nuevas narrativas de la ciudad y del espacio público y de qué manera generan nuevos vínculos de los ciudadanos hacia ella (Sassatelli, 2015). Con el MMVV hemos visto cómo el espacio público se segmenta, se reorganiza y (re)representa gracias y a través de la música. Según nuestro trabajo de campo, el espacio urbano adquiere una nueva identidad y la ciudad, gracias al "teatro» y a los valores que se asocian con el festival, se viste de modernidad y culturalidad, alejándose de su imagen tradicional, proyectándose de manera diferente y desde una posición renovada hacia el exterior. Vemos así cómo los efectos del festival sobre el espacio representado son tanto directos como indirectos. Por un lado, los festivales influyen en las imágenes que se proyectan de la ciudad a través de los medios de comunicación; pero, por el otro, como consecuencia de los impactos sobre la comunidad, influyen sobre la imagen que las personas se hacen de ella. Es decir, los significados que los eventos proyectan sobre el espacio no son solamente historias que los residentes le cuentan al mundo, sino que son también historias que se cuentan a sí mismos.

Esta contribución de los festivales a la creación y transformación del espacio urbano, sin embargo, no está exenta de contradicciones ni tensiones. De hecho, el papel de los festivales en el mapa de agentes y del paisaje urbano público en el contexto actual de interés renovado por la práctica y la espacialidad de las prácticas sociales, de cocreación y relacionalidad, debe familiarizarse y tomar parte en la observación y crítica de las geometrías de poder que se suceden en su uso. Con más razón si son organizados por entidades públicas y tienen lugar en el espacio público. Los residentes son conscientes de los impactos positivos que el festival genera sobre la ciudad, pero también evidencian conflictos de uso que interrumpen negativamente la ciudad. Pero lo que es más, es un festival para la ciudadanía pero sin ella sin - parece ser- mayor consecuencia sobre la gobernanza de la ciudad. Asimismo, las instituciones públicas que organizan el MMVV contribuyen a la privatización de parte de la ciudad y están posicionadas ante una narrativa cultural que legitimiza y apoya los fun- 
damentos comerciales y mercantiles de la música y la cultura. No obstante, gracias a la existencia del festival y alrededor de él se organizan manifestaciones culturales en el espacio público, no ya espontáneas, sino planificadas y con largo recorrido, algunas de ellas clara y pretendidamente al margen del MMVV, que contestan y confrontan las narraciones legitimizadas y autorizadas de la política cultural representada por el mismo MMVV. Estas manifestaciones son generadoras de paisajes culturales extraordinarios y contienen un capital creativo creado en los espacios de conflicto político que utilizan el espacio público como altavoz para proyectar su contestación. Estos agentes también intervienen en la creación y disfrute del ecosistema que se crea alrededor del festival y junto con él participan en un proceso de cocreación líquida del espacio y de sus estructuras de poder. Sin lugar a dudas, estas manifestaciones reconectan el ocio con la acción política, con ese pleasure-politic al que se refería Sharpe (2008) y muestran cómo la ciudad a través de los festivales también se construye en base a la política de los espacios de vida.

\section{Referencias bibliográficas}

Andrew, H. y Leopold, T. (2013). Events and the Social Sciences. Oxon: Routledge. AP, J. (1992). «Residents' perceptions on tourism impacts». Annals of Tourism Research, 19(4), 665-690.

Bailey, C.; Miles, S. y Stark, P. (2004). «Culture led urban regeneration and the revitalisatin of identities in Newcastle Gateshead and the north east of England». International Journal of Cultural Policy, 10, 47-65.

Brennan-Horley, C.; Connell, J. y Gibson, C. (2007). «The Parkes Elvis revival festival: Economic development and con- tested place identities in rural Australia». Geographical Research, 45, 71-84.

DE BRES, K. y DAVIS, J. (2001). «Celebrating group and place identity: A case study of a new regional festival». Tourism Geographies, 3(3), 326-337.

Carlsen, J.; Ali-Knight, J. y Robertson, M. (2007). «Access-A Research Agenda for Edinburgh Festivals». Event Management, 11(1-2), 1-2.

CARMONA, M. (2015). «Re-theorising contemporary public space: a new narrative and a new normative». Journal of Urbanism: International Research on Placemaking and Urban Sustainability, 8(4), 373-405. $<$ http://dx.doi.org/10.1080/17549175.2014.909518>

CUDNY, W. (2014). «Festivals as a subject for geographical research». Geografisk Tidsskrift-Danish Journal of Geography, 114(2), 132-142. <https://doi.org/10.1080/00167223.2014.895673>

CURTIS, R. A. (2010). «Australia's capital of Jazz? The (re)crea-tion of place, music and community at the Wangaratta Jazz festival». Australian Geographer, 41, 101-116.

DeErY, M.; JAGO, L. y FredLINE, L. (2012). «Rethinking social impacts of tourism research: A new research agenda». Tourism Management, 33(1), 64-73. $<$ http://dx.doi.org/10.1016/j.tourman.2011.01.026>

Delamere, T.; Wankel, L. y Hinch, T. (2001). «Development of a scale to measure resident attitudes toward the social impacts of community festivals, part I: item generation and purification of the measure». Event Management, 7, 11-24. 
DERrET, R. (2003). «Making sense of how festivals demonstrate a community's sense of place». Event Management, 8(1), 49-58.

Dredge, D. y Whitford, M. (2011). «Event tourism governance and the public sphere». Journal of Sustainable Tourism, 19(4-5), 479-499. $<$ https://doi.org/10.1080/09669582.2011.573074>

DWYER, L. et al. (2000). "Forecasting the economic impacts of events and conventions». Event Management, 6, 191-204.

Evans, G. (2005). «Measure for measure: Evaluating the evidence of culture's contribution to regeneration». Urban Research, 42, 959-983.

FAULKNER, B. et al. (2000). "Monitoring the tourism impacts of the Sydney 2000 olympics». Event Management, 6(4), 231-246.

Fredline, L.; JaGO, L. y DEERY, M. (2003). «The development of a generic scale to measure the social impacts of events». Event Management, 8, 23-37.

GETZ, D. (2007). Event studies: Theory, research and policy for planned events. Elsevier, ed., Oxford: Butterworth Heinemann.

Getz, D. y PAGE, S. (2015). «Progress and prospects for event tourism research». Tourism Management. <https://doi.org/10.1016/j.tourman.2015.03.007>

GiBson, C. y Connell, J. (2011). Festival places: Re-vitalizing rural Australia. Bristol: Channel View.

- (2012). Music festivals and regional development in Australia. Farnham: Ashgate.

GiBSON, C. y DAVIDSON, D. (2004). «Tamworth, Australia's "country music capital”: Place marketing, rurality, and resident reactions». Journal of Rural Studies, 20, 387-404.

GiBSON, C. y Homan, S. (2004). «Urban development, live music and public space». International Journal of Cultural Policy, 10(1), 67. <https://doi.org/10.1080/1028663042000212337>

Giovanardi, M.; Lucarelli, A. y Decosta, P. L. (2014). "Co-performing tourism places: The "Pink Night" festival». Annals of Tourism Research, 44, 102-115.

GoTHAM, K. F. (2005). "Theorizing urban spectacles». City, 9(2), 225-246.

Hall, M. y Page, S. (2012). "Geography and the study of events». En: S. PaGE y J. CONNELl (eds.). Routledge hand-book of events. Oxon: Routledge, 148-164.

Higham, J. y Ritchie, B. (2001). "The evolution of festivals and other events in rural southern new Zealand». Event Management, 7(1), 39-49.

HitTers, E. (2007). «Porto and Rotterdam as European Capitals of Culture: towards the festivalization of urban cultural policy». En: G. RICHARDS (ed.). Cultural Tourism: Global and local perspectives. Bonghampton: Haworth Press, 281-301.

JONES, M. (2009). «Phase space: geography, relational thinking, and beyond». Progress in Human Geography, 33(4), 487-506.

LARSEN, J. (2011). "Performance, space and tourism». En: J. WILSON (ed.). The Routledge Handbook of Tourism Geographies. Oxon: Routledge, 67-73.

LefebVRE, H. (2013). La producción del espacio. Madrid: Capitán Swing Libros.

LUCAS, M. y WRIGHT, A. (2013). "Liminal performativity: Modern-day festivals and embodied practice». En: European Group for Organization Studies Colloquium (EGOS 2013), 5-7 de julio de 2013, Montreal, Canadá.

MalPAS, J. (2015). «Pensar topográficamente: lugar, espacio y geografía». Documents d'Anàlisi Geogràfica, 61(2), 199-229. <https://doi.org/10.5565/rev/dag.297>

MARKWELL, K. y WAITT, G. (2009). «Festivals, space and sexuality: gay pride in Australia». Tourism Geographies, 11(2), 143-168. 
Massey, D. (2005). For Space. Londres: Sage Publications.

MosCARDO, G. (2007). "Analyzing the role of festival and events in regional development». Event Management, 11, 23-32.

O'Sullivan, D.; Pickernella, D. y SenYardb, J. (2009). «Public sector evaluation of festivals and special events». Journal of Policy Research in Tourism, Leisure and Events, 1 (1), 19-36.

Ortiz, A.; Prats, M. y Baylina, M. (2014). «Procesos de apropiación adolescente del espacio público: otra cara de la renovación urbanística en Barcelona». Boletín de la Asociación de Geógrafos Españoles, 65, 37-58.

Presbury, R. y Edwards, D. (2005). "Incorporating Sustainability in Meetings and Event Management Education». International Journal of Event Management Research, 1 (1), 30-45.

QuinN, B. (2006). "Problematising "festival tourism": Arts festivals and sustainable development in Ireland». Journal of Sustainable Tourism, 14 (3), 288-306. <https://doi.org/10.1080/09669580608669060>

Richards, G. y Palmer, R. (2010). Eventful cities. Cultural management and urban revitalization. Oxford: Elsevier.

RodÓ-DE-ZÁrATE, M. (2013). «Gènere, cos i sexualitat. La joventut, l'experiència i l'ús de l'espai públic urbà». Papers, 98 (1), 127-142.

Russo, A. P. (2013). «Las nuevas fronteras del estudio del turismo: retos conceptuales y epistemológicos tourism: conceptual and epistemological challenges». Revista CIDOB, 113, 15-32.

SASSATELli, M. (2015). "Festivals, urbanity and the public sphere: reflections on European Festivals». En: C. Newbold et al. (eds.). Focus on Festivals. Contemporary European Case Studies and perspectives. Oxford: Goodfellow Publishers, Ltd, 28-39.

SHARPE, E. K. (2008). «Festivals and Social Change: Intersections of Pleasure and Politics at a Community Music Festival». Leisure Sciences, 30 (3), 217-234.

SHARPLEY, R. (2014). «Host perceptions of tourism: A review of the research». Tourism Management, 42, 37-49. <https://doi.org/10.1016/j.tourman.2013.10.007>

SMALL, K. (2008). «Social dimensions of community festivals: an application of factor analysis in the development of the social impact perception (sip) scale». Event Management, 11 (1-2), 45-55.

SMALL, K.; EDWARS, D. y SHERIDAN, L. (2005). «A flexible framework for evaluating the socio-cultural impacts of a (small) festival». International Journal of Event Management Research, 1 (1), 66-77.

Stevens, Q. y Shin, H. (2014). "Urban Festivals and Local Social Space». Planning Practice, 29 (1), 37-41.

Thrift, N. (2006). Space. Theory, Culture \& Society, 23(2-3), 139-146.

Villarino, B. E. (2012). «La idea de espacio público en Geografía Humana. Hacia una conceptualización (crítica) contemporánea». Documents d’Anàlisi Geogràfica, 58 (1), 137-163.

$<$ https://doi.org/10.5565/rev/dag.200>

WAITT, G. y STAPEL, C. (2011). "“Fornicating on floats”? The cultural politics of the Sydney Mardi Gras Parade beyond the metropolis». Leisure Studies, 30 (2), 197-216. <https://doi.org/10.1080/02614367.2010.509445>

WEAVER, D. B. y LAWTON, L. J. (2013). «Resident perceptions of a contentious tourism event». Tourism Management, 37, 165-175. <http://dx.doi.org/10.1016/j.tourman.2013.01.017> 